\title{
HBV Genotype Assay
}

National Cancer Institute

\section{Source}

National Cancer Institute. HBV Genotype Assay. NCI Thesaurus. Code C117108.

An assay used to determine which hepatitis B virus genome(s) is present in an infection. Seven genotypes of HBV (designated A through G) have been identified. Different genotypes are prevalent in different geographical areas and each carries a different risk of development of hepatocellular carcinoma. 